\title{
The Molecular Structure of Monomeric Bis(neopentyl)magnesium by Gas Phase Electron Diffraction
}

\author{
E. C. ASHBY, ${ }^{a}$ LIV FERNHOLT, ${ }^{b}$ ARNE HAALAND, ${ }^{b, *}$ RAGNHILD SEIP $^{\mathrm{b}}$ and \\ R. SCOTT SMITH ${ }^{\mathrm{a}}$
}

a School of Chemistry, Georgia Institute of Technology, Atlanta, Georgia 30332, U.S.A. and

${ }^{b}$ Department of Chemistry, University of Oslo, Blindern, Oslo 3, Norway

The electron scattering pattern of gaseous bis(neopentyl)magnesium recorded with a reservoir pressure of about 1 Torr and a nozzle temperature of about $160{ }^{\circ} \mathrm{C}$, is consistent with monomeric species with $\angle \mathrm{CMgC}=180^{\circ}$. The most important structure parameters are $r_{\mathrm{a}}(\mathrm{Mg}-\mathrm{C})=2.126(6) \AA, l(\mathrm{Mg}-\mathrm{C})$ $=0.086(5) \AA, \angle \mathrm{MgCC}=118.3(1.2)^{\circ}$ and $r_{\mathrm{a}}(\mathrm{C}-\mathrm{C})$ $=1.541(2) \AA$.

Dimethyl- and diethyl-magnesium are white solid substances with exceptionally low volatility and solubility in noncomplexing solvents. ${ }^{1}$ Their crystal structures have been determined by E. Weiss. ${ }^{2,3}$ Both compounds consist of infinite straight chains of $\mathrm{Mg}$ atoms, each surrounded by four bridging alkyl groups. The $\mathrm{Mg}-\mathrm{C}_{\text {bridge }}$ bond distance is $2.24 \pm 0.03 \AA$ in dimethylmagnesium ${ }^{2}$ and $2.26 \pm$ $0.05 \AA$ in diethylmagnesium. ${ }^{3}$

Recently Anderson and Wilkinson reported the synthesis of bis(beopentyl)magnesium. ${ }^{4}$ The compound is freely soluble in benzene, in which it is trimeric, and it sublimes at $110{ }^{\circ} \mathrm{C}$ in vacuum.

Since no structural information is available for.a

- monomeric non-complexed dialkylmagnesium compound, and since we have found it possible to determine the molecular structures of monomeric dimethylberyllium ${ }^{5}$ and trimethylaluminium ${ }^{6}$ by gas phase electron diffraction, using nozzle temperatures of about 160 and $215^{\circ} \mathrm{C}$, respectively, we decided to investigate bis(neopentyl)magnesium in order to fill the gap.

\section{EXPERIMENTAL}

Synthesis. The bis(neopentyl)magnesium was prepared by the method according to Wilkinson ${ }^{4}$ with

* To whom correspondence should be addressed.

0302-4377/80/030213-05\$02.50

(C) 1980 Acta Chemica Scandinavica the following modifications: (1) Neopentyl bromide was used to prepare the Grignard, (2) the precipitation of $\mathrm{MgBr}_{2}$ as the bis dioxanate was affected using a $0.2 \mathrm{M}$ solution of neopentylmagnesium bromide for which standardization for magnesium and bromine had been performed, (3) only a $3 \%$ molar excess of dioxane over that necessary to quantitatively form $\mathrm{MgBr}_{2}$ bis dioxanate was used.

The bis(neopentyl)magnesium in ether was desolvated under vacuum at room temperature overnight to give a viscous liquid, which was transferred to a sublimation apparatus. The viscous liquid was heated in vacuo $\left(10^{-4}\right.$ Torr) at $90{ }^{\circ} \mathrm{C}$. Overnight a white solid collected on the cold finger, which was resublimed for 3 days at $80^{\circ} \mathrm{C}\left(10^{-4}\right.$ Torr). The white crystalline solid collected on the cold finger gave a wt. $\% \mathrm{Mg}$ analysis of $14.4 \%$ (calculated for bis(neopentyl)magnesium $14.65 \%$ ). The NMR in $\mathrm{C}_{6} \mathrm{H}_{6}$ at ambient temperature gave two singlets at $-5.93 \mathrm{ppm}$ and $-6.92 \mathrm{ppm}$ from $\mathrm{C}_{6} \mathrm{H}_{6}$ with an integration of $8.9 / 2$. In addition, dioxane, amounting to $2 \mathrm{~mol} \%$, was identified by NMR.

Diethyl ether (Fisher Scientific) was predried over Nawire and distilled from $\mathrm{NaAlH}_{4}$ under nitrogen. Dioxane was distilled under nitrogen from $\mathrm{CaH}_{2}$. Neopentyl alcohol (Aldrich $98 \%$ ) was used without further purification. Neopentyl bromide was prepared by the method of Hershikowitz ${ }^{7}$ and purified by washing with cold concentrated $\mathrm{H}_{2} \mathrm{SO}_{4}$, saturated $\mathrm{NaHCO}_{3}$ solution, $\mathrm{H}_{2} \mathrm{O}$, and then distilled under nitrogen from $\mathrm{CaH}_{2}$.

Electron diffraction data. The electron scattering pattern of bis(neopentyl)magnesium was recorded on Balzers Eldigraph KDG-2 with reservoir and nozzle temperatures of about $160{ }^{\circ} \mathrm{C}$.

In order to keep the temperature as low as possible and thus keep thermal decomposition at a minimum, we used a modification of the normal 
Table 1. Root mean square vibrational amplitudes (in $\AA$ ) of benzene at ambient temperature. A: Average of six sets of plates obtained with wide beam nozzle. B: Average of six sets of plates obtained with normal nozzle. C: Values from column A corrected by eqn. (1). D: Calculated from molecular force field. ${ }^{9}$

\begin{tabular}{llllll}
\hline & $\mathrm{A}$ & $\mathrm{B}$ & $\mathrm{C}$ & $\mathrm{D}$ & $r_{\mathrm{a}} / \AA$ \\
\hline$l(\mathrm{C}-\mathrm{H})$ & $0.0767(10)$ & $0.0729(7)$ & $(0.0745(10))$ & 0.0771 & 1.10 \\
$l(\mathrm{C}-\mathrm{C})$ & $0.0457(6)$ & $0.0447(4)$ & $(0.0429(6))$ & 0.0464 & 1.40 \\
$l\left(\mathrm{C}_{1}-\mathrm{C}_{3}\right)$ & $0.0615(6)$ & $0.0573(7)$ & $0.0567(6)$ & 0.0552 & 2.42 \\
$l\left(\mathrm{C}_{1}-\mathrm{C}_{4}\right)$ & $0.0690(9)$ & $0.0622(7)$ & $0.0634(9)$ & 0.0590 & 2.79 \\
$l\left(\mathrm{C}_{1}-\mathrm{H}_{2}\right)$ & $0.1020(11)$ & $0.0979(5)$ & $0.0977(11)$ & 0.0998 & 2.16 \\
$l\left(\mathrm{C}_{1}-\mathrm{H}_{3}\right)$ & $0.1044(8)$ & $0.0973(11)$ & $0.0976(8)$ & 0.0967 & 3.40 \\
\hline
\end{tabular}

scattering geometry suggested by A. A. Ivanov at Moscow State University ${ }^{8}$ which permits the scattering pattern to be recorded with a reservoir pressure of about 1 Torr as opposed to about 10 Torr for a normal geometry. The electron beam is convergent and focused at the center of the photographic plate. At the height of the gas nozzle the diameter is about $2 \mathrm{~mm}$. The nozzle opening is rectangular with a width of $4.0 \mathrm{~mm}$ and a height of $0.6 \mathrm{~mm}$, but otherwise the inlet system is of conventional design.

The modified geometry represents a greater deviation from a point-like scattering region. At larger scattering angles the convergent beam will no longer be focused at the photographic plate and a certain blurring of the diffraction pattern must be expected. A conventional structure refinement may then yield vibrational amplitudes, $l$ values, which are too large. The blurring of the diffraction pattern should be most effective for those terms in the molecular intensity which vary most rapidly with the diffraction angle, i.e. those terms representing the larger internuclear distances. If detectable, we expect the systematic error introduced into the vibrational amplitudes by the unconventional scattering geometry to increase with increasing internuclear distance $r_{\mathrm{a}}$.

Till now we have recorded six sets of plates, each consisting of at least four plates, with wide beam geometry and with benzene as a test substance. The nozzle-to-plate distance was $25 \mathrm{~cm}$ in each case. In Table 1 we compare average values for the vibrational amplitudes obtained by standard refinement procedures with average values obtained from six plate sets obtained with normal scattering geometry and with amplitudes calculated from a molecular force field. ${ }^{9}$ It is seen that in every case the wide beam geometry yields slightly larger vibrational amplitudes than the conventional geometry. The largest difference, $0.0071(14) \AA$ is obtained for the longest internuclear distance, $\mathrm{C}_{1}-\mathrm{H}_{3}=3.40 \AA$. It may be noted, however, that in the case of the bond distances, the wide beam values are actually in better agreement with the values calculated from the molecular force field.

In Table 1 we also list the values obtained for the vibrational amplitudes of the larger, non-bonded distances by applying the correction (1).

$l_{\text {corr }}=h_{\text {uncorr }}-0.002 r_{\mathrm{a}}$

It is seen that this correction leads to excellent agreement between the amplitudes obtained by wide beam and conventional geometry, and to better agreement with the $l$ values calculated from a molecular force field.

Until more information is available, we shall therefore adopt the following - somewhat arbitrary - procedure when reporting vibrational amplitudes based on wide beam data: The amplitudes of $\mathrm{C}-\mathrm{H}$ and $\mathrm{C}-\mathrm{C}$ bond distances are left unchanged, while all other amplitudes are corrected according to eqn. (1).

Data processing. The scattering pattern from bis(neopentyl)magnesium was recorded with nozzleto-plate distances of 50 and $25 \mathrm{~cm}$. The optical densities of three plates from each set were processed using the programs described by Andersen et al. ${ }^{10}$ The modified molecular intensity points obtained by averaging the intensity values for each nozzle-to-plate distance are shown in Fig. 1.

\section{STRUCTURE REFINEMENT}

It was assumed that an adequate model of bis(neopentyl)magnesium can be construced from two neopentane units of $T_{\mathrm{d}}$ symmetry by removing one $\mathrm{H}$ atom from each and replacing it by the $\mathrm{Mg}$ atom. The $\angle \mathrm{MgCC}$ valence angle was allowed to vary, while the plane of the methylene group was assumed to bisect $\angle \mathrm{MgCC}$. The $\angle \mathrm{HCH}$ angle of the methylene group was fixed at $109.5^{\circ}$. Finally it 

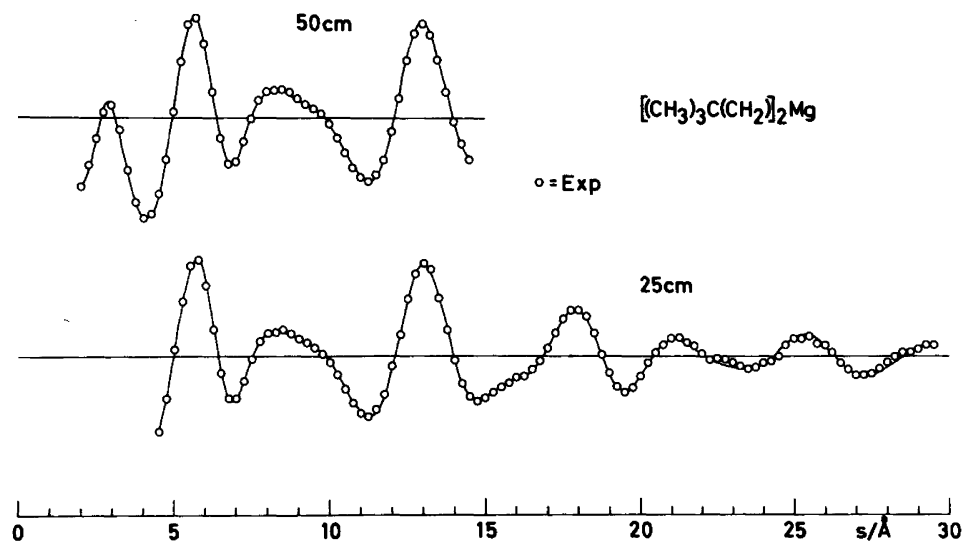

Fig. 1. O: Experimental modified intensity points from $s=2.00$ to $14.50 \AA^{-1}$ (upper curve) and from $s=4.50$ to $29.50 \AA^{-1}$ (lower curve). In the upper curve only every second experimental point is shown. Full lines: Theoretical intensity curves calculated for best model.

was assumed that $\angle \mathrm{CMgC}$ is $180^{\circ}$. Under the foregoing assumptions the structure of bis(neopentyl)magnesium is determined by six independent parameters, for instance the $\mathrm{C}-\mathrm{H}, \mathrm{C}-\mathrm{C}$ and $\mathrm{Mg}-\mathrm{C}$ bond distances, the $\angle \mathrm{MgCC}$ and $\angle C(2) C(3) H$ valence angles and a dihedral angle $\phi$ defining the relative orientation of the two neopentyl groups. A molecular model with $\phi=180^{\circ}$, i.e. with the neopentyl groups anti, is shown in Fig. 2.

An experimental radial distribution curve obtained by Fourier inversion of the connected molecular intensity curves is shown in Fig. 3. All peaks can be assigned to interatomic distances in the monomer as indicated by vertical bars. It is seen that while distances within each $\mathrm{Mg}\left(\mathrm{CH}_{2}\right) \mathrm{C}\left(\mathrm{CH}_{3}\right)_{3}$ fragment are represented by clearly defined peaks

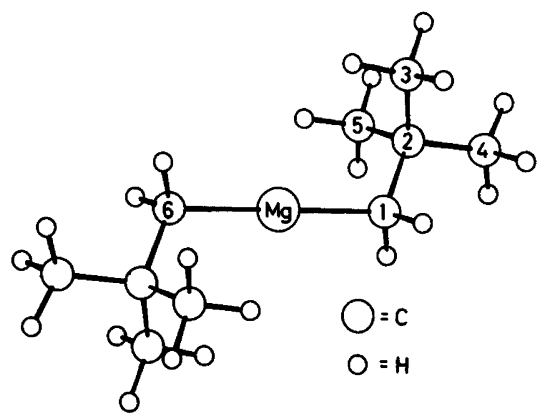

Fig. 2. Molecular model of bis(neopentyl)magnesium. The hydrogen atoms are numbered according to the $\mathrm{C}$ atoms to which they are bonded. in the RD curve, the curve contains no such peak that can be assigned to distances between atoms in different neopentyl groups. It seems reasonable to assume that these groups undergo virtually nonhindered internal rotation about the $\mathrm{CMgC}$ axis.

Initial backgrounds were drawn using theoretical intensities calculated for the anti model. Distance vectors between atoms in different neopentyl groups were assigned vibrational amplitudes of the order of $0.5 \AA$. The dihedral angle was refined along with the other independent parameters, but no significance should be attached to the value obtained $\left(140^{\circ}\right)$.

The six independent structure parameters and eight root mean square vibrational amplitudes were refined by least-squares calculations on the intensity data under the constraints of a geometrically consistent $r_{\mathrm{a}}$ structure with a non-diagonal weight matrix. ${ }^{11}$ The parameter values obtained are listed in Table 2. All vibrational amplitudes except those of the $\mathrm{C}-\mathrm{H}$ and $\mathrm{C}-\mathrm{C}$ bond distances have been corrected according to eqn. (1).

\section{DISCUSSION}

The $\mathrm{Mg}-\mathrm{C}$ bond distance in bis(neopentyl)magnesium, $2.126(5) \AA$, is $0.17 \AA$ larger than the $\mathrm{Al}-\mathrm{C}$ bond distance in $\left(\mathrm{CH}_{3}\right)_{3} \mathrm{Al}^{6}$ and $0.25 \AA$ larger than the $\mathrm{Si}-\mathrm{C}$ bond distance in $\left(\mathrm{CH}_{3}\right)_{4} \mathrm{Si} ;{ }^{12}$ the increase of atomic radius on going from right to left in the periodic table is evident. 


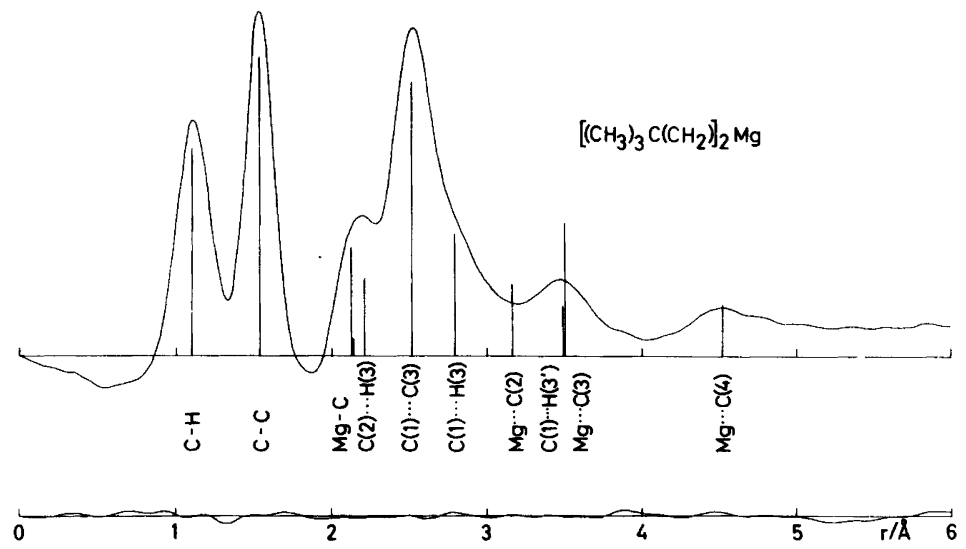

Fig. 3. Upper curve: Experimental radial distribution curve for bis(neopentyl)magnesium. Between $r=6$ and $9 \AA$ the curve decreases slowly to zero, but contains no significant features. Lower curve: Difference between the experimental curve and a theoretical curve calculated for best model. Artifical damping constant, $k=0.002 \AA^{2}$.

The metal-carbon bond distances of $\left(\mathrm{CH}_{3}\right)_{2} \mathrm{Be}$ and $\left(\mathrm{CH}_{3}\right)_{3} \mathrm{Al}$ increase on formation of complexes with electron donors. ${ }^{13} \mathrm{~A}$ similar effect is found in

Table 2. Bond distances, valence angles and root mean square vibrational amplitudes of bis(neopentyl)magnesium. (Estimated standard deviations in parenthesis. $)^{a}$

\begin{tabular}{lll}
\hline & $r_{\mathrm{a}} / \AA$ & $l / \AA^{b}$ \\
\hline $\mathrm{Mg}-\mathrm{C}$ & $2.126(6)$ & $0.086(5)$ \\
$\mathrm{C}-\mathrm{C}$ & $1.541(2)$ & $0.059(2)$ \\
$\mathrm{C}-\mathrm{H}$ & $1.105(2)$ & $0.072(2)$ \\
& & \\
$\mathrm{Mg}-\mathrm{C}(2)$ & $3.162(18)$ & $0.191(32)$ \\
$\mathrm{Mg}-\mathrm{C}(3)$ & $3.502(26)$ & $0.343(27)$ \\
$\mathrm{Mg}-\mathrm{C}(4)$ & $4.519(12)$ & $0.176(13)$ \\
$\mathrm{C}(1)-\mathrm{C}(3)$ & $2.516(3)$ & $0.086(2)^{\mathrm{c}}$ \\
$\mathrm{C}(1)-\mathrm{C}(6)$ & $4.251(12)$ & 0.150 (ass $\left.^{b}\right)$ \\
$\mathrm{C}(2)-\mathrm{H}(3)$ & $2.212(9)$ & $0.126(2)^{c}$ \\
$\mathrm{C}(1)-\mathrm{H}(3)$ & $2.792(11)$ & $0.191(10)^{d}$ \\
$\mathrm{C}(1)-\mathrm{H}(3)$ & $3.500(7)$ & $0.130(10)^{d}$ \\
& & \\
$\angle \mathrm{CMgC}=180^{\circ}($ ass) & \\
$\angle \mathrm{MgCC}=118.3(1.2)^{\circ}$ & \\
$\angle \mathrm{CCC}=109.5^{\circ}($ ass $)$ & \\
$\angle \mathrm{CCH}(3)=112.4(0.7)^{\circ}$ & \\
\hline
\end{tabular}

${ }^{a}$ For numbering of the atoms consult Fig. 2. ${ }^{b}$ All amplitudes except $l \mathrm{C}-\mathrm{C})$ and $l(\mathrm{C}-\mathrm{H})$ have been corrected according to eqn. (1). ${ }^{c, d}$ These amplitudes were assumed to differ by the same amount as in neopentane. $^{16}$ organomagnesium compounds. The two crystallographically nonequivalent $\mathrm{Mg}-\mathrm{C}$ bond distances in the 1:2 complex of $\left(\mathrm{CH}_{3}\right)_{2} \mathrm{Mg}$ with quinuclidine are 2.169(9) and 2.224(8) $\AA$, respectively; ${ }^{14}$ and the two nonequivalent $\mathrm{Mg}-\mathrm{C}$ distances in the complex of diphenylmagnesium with tetramethylethylenediamine are both $2.167(3) \AA{ }^{15}$ even though the covalent radius of $s p^{2}$ hybridized $\mathrm{C}$ is assumed to be about $0.03 \AA$ less than the radius of $s p^{3}$ hybridized $\mathrm{C}$. The $\angle \mathrm{CMgC}$ valence angles in the two complexes are $142^{\circ}$ and $119^{\circ}$, respectively.

The vibrational amplitude obtained for the $\mathrm{C}-\mathrm{C}$ bond distance is $0.008 \AA$ larger than in neopentane. ${ }^{16}$ $l=0.051\left(1_{3}\right) \AA$, and the amplitude obtained for the $\mathrm{C}(1)-\mathrm{C}(3)$ distance is $0.015 \AA$ greater than in neopentane, $0.071(1) \AA$. We believe the large values to be due to deviations from the high local symmetry imposed on the model. The $\mathrm{C}-\mathrm{C}$ bond distance obtained must therefore be regarded as an average value.

The vibrational amplitude of the $\mathrm{Mg}-\mathrm{C}$ bond distance, $l=0.086(5) \AA$, is considerably larger than the $\mathrm{Al}-\mathrm{C}$ amplitude in $\left(\mathrm{CH}_{3}\right)_{3} \mathrm{Al}^{6}, l=0.061(1) \AA$, or the $\mathrm{Si}-\mathrm{C}$ amplitude in $\left(\mathrm{CH}_{3}\right)_{4} \mathrm{Si}^{1{ }^{12}} \mathrm{l}=0.053(5)$ $\AA$. We believe the large amplitude to be due to the increased polarity of the metal carbon bond as well as to the increased size of the metal atom.

Acknowledgement. We are grateful to the Norwegian Research Council for Science and the Humanities for financial support. 


\section{REFERENCES}

1. Coates, G. E., Green, M. L. H. and Wade, K. Organometallic Compounds, Methuen, London 1967.

2. Weiss. E. J. Organomet. Chem. 2 (1964) 314.

3. Weiss. E. J. Organomet. Chem. 4 (1965) 101.

4. Andersen, R. A. and Wilkinson, G. J. Chem. Soc. Dalton Trans. (1977) 809.

5. Almenningen, A., Haaland, A. and Morgan, G. L. Acta Chem. Scand. 23 (1969) 2921.

6. Almenningen, A., Halvorsen, S. and Haaland, A. Acta Chem. Scand. 25 (1971) 1937.

7. Wiley, G. A., Hershkowitz, R. L., Rein, B. M. and Chung, B. C. J. Am. Chem. Soc. 86 (1964) 964.

8. Ivanov, A. A. and Zasorin, E. Z. Prib. Tekh. Eksp. In press; Annual Report of the Norwegian Electron Diffraction Group, 1979.

9. Cyvin, S. J. Molecular Vibrations and Mean Square Amplitudes, Oslo, Universitetsforlaget 1968.

10. Andersen, B., Seip, H. M., Strand, T. G. and Stølevik, R. Acta Chem. Scand. 23 (1969) 23.

11. Seip, H. M., Strand, T. G. and Stølevik, R. Chem. Phys. Lett. 3 (1969) 617.

12. Beagley. B.. Monaghan. J. J. and Hewitt. T. G. J. Mol. Struct. 8 (1971) 401.

13. Haaland, A. Topics in Current Chemistry 53 (1975) 1.

14. Toney, J. and Stucky, G. D. J. Organomet Chem. 22 (1970) 241.

15. Spek, A. L., Schat, G., Holtkamp, H. C., Blomberg, C. and Bickelhaupt, F. J. Organomet. Chem. 131 (1977) 331.

16. Bartell, L. S. and Bradford, W. F. J. Mol. Struct. 37 (1977) 113.

Received November 26, 1979. 